\title{
Shentong Zhuyu Decoction Inhibits Inflammatory Response, Migration, and Invasion and Promotes Apoptosis of Rheumatoid Arthritis Fibroblast-like Synoviocytes via the MAPK p38/PPAR $\gamma /$ CTGF Pathway
}

\author{
Ying Han, ${ }^{1,2}$ Jing Wang, ${ }^{3}$ Meng Jin, ${ }^{2}$ Lin Jia, ${ }^{4}$ Cuihuan Yan, ${ }^{5}$ and Yali Wang ${ }^{1}$ \\ ${ }^{1}$ College of Integrated Chinese and Western Medicine, Hebei Medical University, Shijiazhuang, China \\ ${ }^{2}$ Department of Chinese Medicine, The Third Hospital of Hebei Medical University, Shijiazhuang, China \\ ${ }^{3}$ Department of Chinese Medicine Diagnostics, Hebei University of Chinese Medicine, Shijiazhuang, China \\ ${ }^{4}$ Department II of Respiratory, Hebei Provincial Hospital of Traditional Chinese Medicine, Shijiazhuang, China \\ ${ }^{5}$ Institute of Integrated Chinese and Western Medicine, Hebei University of Chinese Medicine, Shijiazhuang, China
}

Correspondence should be addressed to Yali Wang; lvyihongni1988@163.com

Received 17 August 2020; Revised 23 December 2020; Accepted 29 December 2020; Published 16 January 2021

Academic Editor: Marta Laranjo

Copyright $\odot 2021$ Ying Han et al. This is an open access article distributed under the Creative Commons Attribution License, which permits unrestricted use, distribution, and reproduction in any medium, provided the original work is properly cited.

Introduction. The current study is aimed at exploring the effect of Shentong Zhuyu Decoction on the proliferation, migration, invasion, and apoptosis of rheumatoid arthritis fibroblast-like synoviocytes (RA-FLS) and its underlying molecular mechanism. Materials and Methods. The type II collagen-induced arthritis (CIA) model was established. Subsequently, the RA-FLS were isolated from the CIA rat model and identified by immunohistochemistry. The viability, apoptosis, cell cycle, migration, and invasion of RA-FLS were detected by the cell counting kit 8 (CCK-8) assay, flow cytometry, wound-healing assay, and transwell invasion assay, respectively. The levels of MAPK p38, PPAR $\gamma$, CTGF, Bcl-2, Bax, caspase-3, IL-1 $\beta$, MMP-3, CDK4, and cyclin D1 were determined by qRT-PCR and western blotting, respectively. Results. After treatment with Shentong Zhuyu Decoction medicated serum, the $\mathrm{OD}_{570}$ value, migrative and invasive abilities, and the secretion of IL- $1 \beta$, MMP-3 were remarkably decreased in RA-FLS, while the apoptosis rate was increased. Further, results showed that Shentong Zhuyu Decoction inhibited the transition from the G1 phase to S phase. Additionally, Shentong Zhuyu Decoction significantly inhibited the expression of Bcl-2, CDK4, cyclin D1, MAPK p-p38, and CTGF, whereas elevated the levels of Bax, caspase-3, and PPAR $\gamma$. Importantly, the effects of Shentong Zhuyu Decoction were consistent with the trends of MAPK P38 inhibitor (SB203580) and PPAR $\gamma$ agonist (GW1929). Conclusions. Shentong Zhuyu Decoction inhibited viability, inflammatory response, migration, invasion, and transition from the G1 phase to S phase and promoted apoptosis of RA-FLS via the MAPK p38/PPAR $\gamma /$ CTGF pathway.

\section{Introduction}

Rheumatoid arthritis (RA) is a chronic autoimmune disease with high prevalence and disability, which involves in multiple joints throughout the body $[1,2]$. Currently, the treatment of RA is mainly based on drugs and surgeries [3]. However, high costs seriously trouble the patients, and treatments are far from meeting the needs of RA patients. Furthermore, the pathology of RA is still unclear [4]. As we known, fibroblast-like synoviocytes (FLS) show tumor-like characteristics, which are considered to be the "leader cells" in terms of promoting the persistence and destructiveness in disease. Moreover, FLS play a major role on recurrence, long-term cure, and poor prognosis of RA $[5,6]$. Previous research has found that targeted treatment for FLS improves the condition of RA and indicated that direct regulation of FLS may become the next target for RA treatment [7]. Therefore, exploring the underlying mechanism and seeking for new therapy are urgent for public health. 
Previous study has indicated that the mitogen-activated protein kinase (MAPK) family members play vital roles on progression of RA including MAPK p38, extracellular signal-regulated kinase (ERK), and c-Jun N-terminal kinase (JNK). In particular, MAPK p38 is involved in the upregulation of proinflammatory cytokines [8]. Moreover, the MAPK p38 pathway has been proved to participate in the activation of RA-FLS $[9,10]$. Peroxisome proliferator-activated receptors (PPARs) are known as ligand-activated transcription factors. In terms of RA, a previous study has demonstrated that activation of PPAR $\gamma$ can effectively suppress the inflammatory response [11]. In addition, connective tissue growth factor (CTGF) is a downstream molecule of PPAR $\gamma$ [12] and plays a critical role on RA progression, which is verified, respectively [13]. Furthermore, a previous study has indicated that activation of MAPK p38 negatively regulates PPAR $\gamma$ [14]. Therefore, we hypothesized that the MAPK p38/PPAR $\gamma /$ CTGF pathway may involve in RA progression.

More and more evidence have demonstrated that traditional Chinese herbs including liquiritin, berberine, salicin, imperatorin, and paeonol play vital roles on the proliferation, inflammation, and angiogenesis in the RA [15-19]. Furthermore, Duan et al. [20] have indicated the synergistic mechanism of four effective components of compound Fengshiding capsule against RA by network pharmacology, which may provide basis for the further study on compound preparation of traditional Chinese medicine. Shentong Zhuyu Decoction consists of about 12 kinds of traditional Chinese medicine, which plays roles on promoting blood circulation and removing blood stasis [21]. Moreover, previous studies have demonstrated that Shentong Zhuyu Decoction inhibits the inflammatory response and alleviates the pain behaviors $[22,23]$. However, whether Shentong Zhuyu Decoction regulates the molecular mechanism of RA-FLS via the MAPK p38/PPAR $\gamma /$ CTGF pathway has not reported yet.

The current study is aimed at exploring the effect of Shentong Zhuyu Decoction on the proliferation, migration, invasion, and apoptosis of RA-FLS and its underlying molecular mechanism via the MAPK p38/PPAR $\gamma /$ CTGF pathway. Our findings may provide the novel therapy for treated with RA.

\section{Materials and Methods}

2.1. Animal Model. Sprague-Dawley (SD) rats were purchased from Liaoning Changsheng Biotechnology Co., Ltd. (Shenyang, China). The collagen-induced arthritis (CIA) rat model was established as previously described [24]. Briefly, type II collagen (CII, $10 \mathrm{mg}$ ) was dissolved in acetic acid $(0.1 \mathrm{~mol} / \mathrm{L})$ and then mixed with an equal amount of complete Freund's adjuvant (CFA). Subsequently, rats were injected with CII emulsion $(100 \mu \mathrm{L})$ in the root of the tail, respectively. After 7 days, the immunization was repeated again with the same dosage in the same site. The rats were injected with normal saline of equal volume, which is deemed as control. On the $14^{\text {th }}$ days of modeling, a marked swelling of the toe and ankle joint was observed.

The arthritis scores of CIA rats were evaluated by the scoring criteria [25]. In brief, 0 point indicated no erythema and swelling, 1 point indicated mild erythema and swelling of the mid-foot or ankle joint, 2 points indicated mild erythema and swelling extending from the ankle to mid-foot, 3 points indicated moderate swelling extending from the ankle to the metatarsal joints, and 4 points indicated that severe swelling encompass the ankle, foot, and digits. The maximum arthritic score per rats was 16. In general, arthritis scores $>4$ are deemed as an indicator for successful modeling [26]. The paw swelling of CIA rats was assessed by measuring the circumference of the paw, as previously described [27]. Oedema was expressed in percentage compared with the initial control values.

The parameters of the CIA model in the current study indicated that the levels of and rheumatoid factor (RF) and C-reactive protein $(\mathrm{CRP})$ were $(67.43 \pm 13.95) \mathrm{IU} / \mathrm{mL}$ and $(6.72 \pm 1.66) \mathrm{mg} / \mathrm{L}$, paw swelling was $(21.44 \pm 6.00 \%)$, and arthritis score was $8.00 \pm 1.41$. So, the rats with abovementioned parameters were regarded as the successful CIA rat model and then were used for subsequent experiments.

2.2. Prepare the Shentong Zhuyu Decoction. The Shentong Zhuyu Decoction consisted of Gentiana macrophylla (3 g), Ligusticum wallichii (6g), Peach Kernel (9g), Safflower (9g), Licorice (6 g), Notopterygium (3 g), Myrrh (6g), Angelica $(9 \mathrm{~g})$, Trogopterus dung (6g), Rhizoma Cyperi (3g), Achyranthes (9g), Earthworm (6g), Cortex phellodendri (6g), and Rhizoma Atractylodis (6g). Abovementioned herbs were Chinese medicine dispensing granules, which were purchased from Shenwei Pharmaceutical Group Co., Ltd. (Beijing, China). 1 dose of Chinese medicine dispensing granules was equivalent to $87 \mathrm{~g}$ of crude drug, which added boiling water $(55.55 \mathrm{~mL})$ to make the drug concentration of $1.566 \mathrm{~g} / \mathrm{mL}$.

2.3. Medicated Serum Preparation. The dosage of Shentong Zhuyu Decoction was converted by the human and animal body surface area, determining by the common clinical dosage, which was about $15.66 \mathrm{~g} / \mathrm{kg} / \mathrm{d}$. The normal rats were gavaged by Shentong Zhuyu Decoction $(2 \mathrm{~mL})$ and distilled water $(2 \mathrm{~mL})$ for 7 days, respectively. Two hours after the last administration, the rats were anesthetized, and $5 \mathrm{~mL}$ of blood was drawn from the abdominal aorta. The blood was centrifuged at $1000 \times \mathrm{g}$ for $10 \mathrm{~min}$ after stood for $30 \mathrm{~min}$ at room temperature. Following, the drug-containing serum and control serum were collected and stored at $-80^{\circ} \mathrm{C}$. Finally, the drug-containing serum was added into culture medium to detect the effect on RA-FLS, with volume fractions of $5 \%$ (low-dose group), 10\% (medium-dose group), and $20 \%$ (high-dose group), respectively. The appropriate concentration of drug-containing serum was screened using the MTT assay.

2.4. Cell Isolation and Identification. The synovial tissues were taken from normal and CIA rats and cut and digested with DNase $(0.15 \mathrm{mg} / \mathrm{mL})$, hyaluronidase $(0.15 \mathrm{mg} / \mathrm{mL})$, and collagenase $(1 \mathrm{mg} / \mathrm{mL})$. The normal FLS (normal FLS) and RA-FLS were obtained and resuspended with DMEM medium (Gibco, Gaithersburg, MD, USA) containing 10\% fetal bovine serum (FBS, Tianhang Biotechnology Co., Ltd, 
Hangzhou, China), $30 \mathrm{mg} / \mathrm{mL}$ glutamine (Solarbio, Shanghai, China), $2.5 \mu \mathrm{g} / \mathrm{mL}$ amphotericin B (Sigma-Aldrich, St. Louis, Mo, USA), and $10 \mu \mathrm{g} / \mathrm{mL}$ gentamicin (Gibco). After the overnight culture, the nonadherent cells were removed, and adherent cells were routinely cultured.

The RA-FLS were identified by immunohistochemistry. In brief, the cells $\left(2 \times 10^{4}\right.$ cells $\left./ \mathrm{mL}\right)$ were cultured in 12 -well plate for $48 \mathrm{~h}$. Subsequently, cells were fixed with $4 \%$ paraformaldehyde and incubated with $0.1 \%$ TritonX-100 and $3 \%$ hydrogen peroxide. After washed, anti-Vimentin (1: 200, Wanleibio, Shenyang, China) and anti-CD68 (1:200, Affinity Biosciences, OH, USA) were added and incubated overnight at $4^{\circ} \mathrm{C}$. Following, horseradish peroxidase- (HRP-) labeled goat anti-rabbit IgG was added and incubated overnight at $37^{\circ} \mathrm{C}$ for $60 \mathrm{~min}$. Finally, the cells were stained with diaminobenzidine $(\mathrm{DAB})$ and hematoxylin. The images were captured by inverted microscope (BX53, Olympus, Tokyo, Japan).

2.5. Cell Treatment and Grouping. The cells in the logarithmic phase of growth were collected and cultured in 96-well plates $\left(5 \times 10^{3}\right.$ cells $\left./ \mathrm{mL}\right)$. After the cells were adhered, different drugs were intervened for $48 \mathrm{~h}$. The experiment was divided into 5 groups. Among them, normal FLS were cultured in DMEM medium plus 20\% control serum (normal group). RA-FLS were cultured in DMEM medium plus $20 \%$ control serum, P38 mitogen-activated protein kinase inhibitor (MAPK P38 inhibitor, SB203580, $20 \mu \mathrm{mol} / \mathrm{L}$ ), peroxisome proliferator-activated receptor gamma agonist (PPAR $\gamma$ agonist, GW1929, $30 \mu \mathrm{mol} / \mathrm{L}$ ), and 20\% drugcontaining serum, which were named as the blank group, P38 inhibitor group, PPAR $\gamma$ agonist group, and Shentong Zhuyu Decoction group, respectively.

2.6. Cell Viability Assay. Cell viability was detected by the cell counting kit $8(\mathrm{CCK}-8)$ assay. Cells $\left(5 \times 10^{3}\right.$ cells $\left./ \mathrm{mL}\right)$ were seeded in 96-well plates and then intervened with drug for $48 \mathrm{~h}$. CCK-8 solution was added into per well and incubated for $2 \mathrm{~h}$. The $\mathrm{OD}_{570}$ values were measured by microplate reader (ELX-800, Biotek, Winooski, VT, USA).

2.7. Cell Apoptosis Assay. After $48 \mathrm{~h}$ treatment, FLS were collected, washed with cool phosphate buffer saline (PBS) and resuspended. The cell apoptosis was conducted by flow cytometry (NovoCyte, ACEA Bioscience, San Diego, California, USA) using Annexin V-FITC apoptosis detection kit (Wanleibio).

2.8. Cell Cycle Assay. After $48 \mathrm{~h}$ treatment, FLS were collected, fixed with $75 \%$ ethanol, and washed with cool PBS. Subsequently, RNA enzyme-containing propidium iodide (PI) solution was added for incubation. Finally, the cell cycle was detected by instrument (NovoCyte, ACEA Bioscience).

2.9. Wound-Healing Assay. After treatment, FLS were cultured in 6 -well plates $\left(1 \times 10^{5}\right.$ cells $\left./ \mathrm{mL}\right)$ overnight. The wound was obtained by using a $200 \mu \mathrm{L}$ pipette tip on the center of the cell monolayer. Images were captured at $0 \mathrm{~h}$ and $48 \mathrm{~h}$, and the migrative distance was assessed by Image pro plus software.
2.10. Transwell Invasion Assay. The cells $\left(1 \times 10^{5}\right.$ cells $\left./ \mathrm{mL}\right)$ were grown in the top chamber with Matrigel (BD Bioscience, Franklin Lakes, NJ, USA), and DMEM medium was added in the lower chamber. After $48 \mathrm{~h}$, the invasive cells were fixed with $4 \%$ paraformaldehyde and the stained with $0.4 \%$ crystal violet (Amresco, Cleveland, OH, USA). Finally, the amount of cells was calculated via microscope (IX53, Olympus).

2.11. Quantitative Real-Time RT-PCR. Total RNA was extracted from cells using TRIpure isolation reagent (BioTeke, Beijing, China). qRT-PCR analysis was performed as previously described [28]. Briefly, first-strand cDNA was synthesized from total RNA using cDNA synthesis kit (BioTeke). Then, real-time PCR was performed using the SYBR Green kit (Solarbio) and detected by Exicycler TM 96 (Bioneer, Daejeon, Korea). GAPDH was acted as internal control. The primer sequences were synthesized by GenScript Biotechnology (Nanjing, China) and listed as the following: MAPK p38 forward, TAAAGCCCAGCAACCTCG, reverse, CAGCCCACGGACCAAATA; PPAR $\gamma$ forward, TACCAC GGTTGATTTCTC, reverse, AATAATAAGGCGGGGA CG; CTGF forward, GTCTTCGGTGGGTCCGTGTA, reverse, GCGGTCCTTGGGCTCATCAC; CyclinD1 forward, GAGGAGCAGAAGTGCGAAGA, reverse, GGCG GATAGAGTTGTCAGTGTAG; caspase-3 forward, GACG ACAGGGTGCTACGAT, reverse, TTTCCTTACGCTCT GACTGA; $\beta$-actin forward, GGAGATTACTGCCCTGGCT CCTAGC, reverse, GGCGGACTCATCGTACTCCTGCTT. The reaction was conducted with an initial denaturing step at $94^{\circ} \mathrm{C}$ for $5 \mathrm{~min}$, followed by 40 cycles of $94^{\circ} \mathrm{C}$ for $10 \mathrm{~s}$, $60^{\circ} \mathrm{C}$ for $20 \mathrm{~s}$, and $72^{\circ} \mathrm{C}$ for $30 \mathrm{~s}$. The relative expression was analyzed by the $2^{-\Delta \Delta \mathrm{Ct}}$ method.

2.12. Western Blotting. Protein samples were extracted by RIPA buffer, and the concentration was measured by the BCA kit. Protein was separated by SDS-PAGE and then was transferred into polyvinylidene difluoride (PVDF) membrane. After that, membranes were blocked with $5 \%$ skim milk, incubated overnight at $4{ }^{\circ} \mathrm{C}$ with monoclonal anti- $\beta$ actin antibody, anti-p38 antibody, anti-p-p38 antibody, anti-CTGF antibody, anti-MMP-3 antibody, and anti-IL$1 \beta$ antibody (1: 1000, Wanleibio), and then incubated with the horseradish peroxidase- (HRP-) conjugated secondary antibody (1: 10000, Wanleibio) for $50 \mathrm{~min}$. Immunodetection was performed using enhanced chemiluminescence reagent (Wanleibio), and then the protein band was detected using Gel-Pro-Analyzer program (Media Cybernetics, Rockville, MD, USA). $\beta$-actin was used as internal control.

2.13. Statistical Analysis. All data were statistically analyzed with SAS 9.1 software. The measurement data were expressed as mean \pm standard deviation (SD). The differences between two groups were analyzed by the $t$-test. The comparison of multiple groups was calculated with one-way analysis of variance (ANOVA) and followed by the LSD $t$-test. $P<0.05$ was considered as statistically significant. 


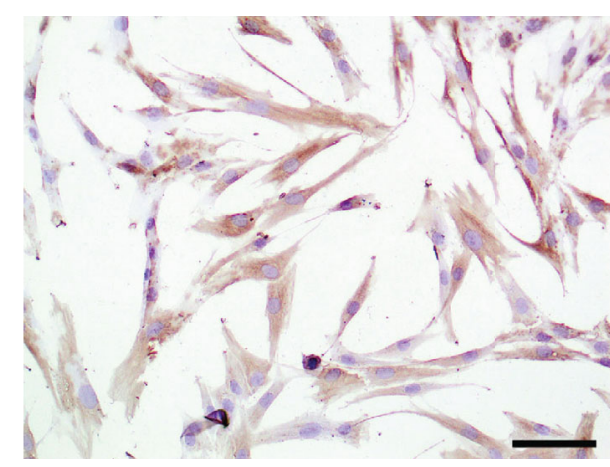

(a)

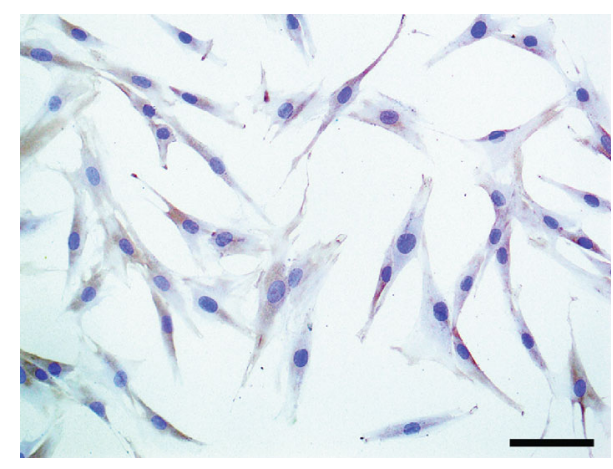

(b)

Figure 1: The rheumatoid arthritis fibroblast-like synoviocytes (RA-FLS) were identified by immunohistochemistry. (a) The results of immunohistochemistry showed that anti-Vimentin antibody was positive $(\times 200)$. (b) The results of immunohistochemistry showed that anti-CD68 antibody was negative $(\times 200)$.

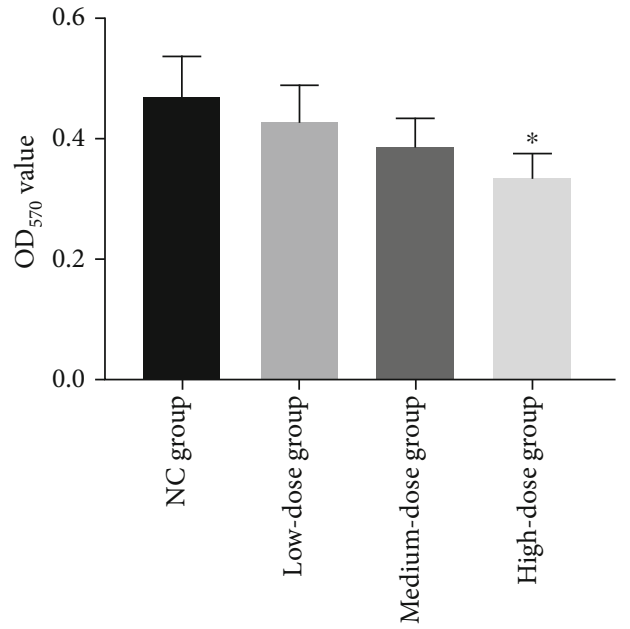

(a)

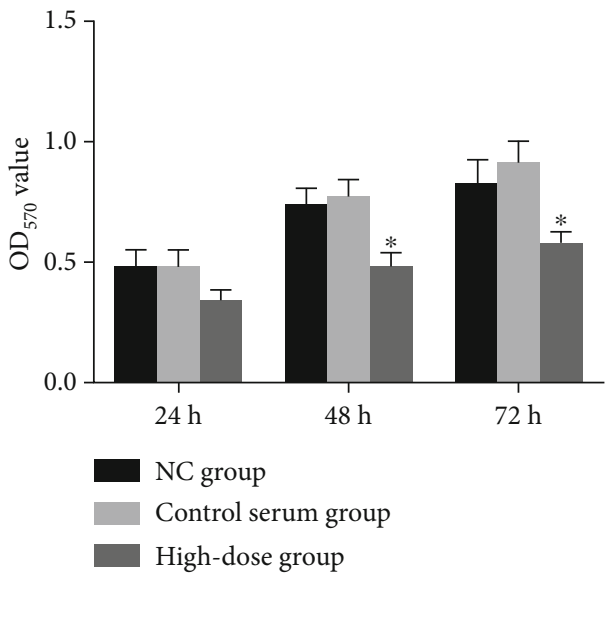

(b)

FIgURE 2: Screening of concentration of Shentong Zhuyu Decoction medicated serum. (a) The $\mathrm{OD}_{570}$ value of different drug-containing serums at $24 \mathrm{~h}$ intervention in RA-FLS. (b) The $\mathrm{OD}_{570}$ value of intervention with drug-containing serum at $24 \mathrm{~h}, 48 \mathrm{~h}$, and $72 \mathrm{~h}$ in $\mathrm{RA}$ FLS. NC group, without any addition in the culture medium; control serum group, culture medium plus $20 \%$ control serum.

\section{Results}

3.1. RA-FLS Identification. After cultured 3 generations, RAFLS presented typically fibroblast-like morphology, which was characterized by elongated spindle-shaped, elongated protrusions, nucleus-centered, round, or oval shape and arrangement of polarity. The results of immunohistochemistry showed that anti-Vimentin antibody was positive, and anti-CD68 antibody was negative (Figures 1(a) and 1(b)). Abovementioned results were in accordance with the characteristics of RA-FLS.

\subsection{Screening of Concentration of Shentong Zhuyu Decoction} Medicated Serum. MTT results showed that the $\mathrm{OD}_{570}$ value was significantly decreased in the high-dose group compared with the NC group $(P<0.05)$, indicating that intervention with $20 \%$ drug-containing serum for $24 \mathrm{~h}$ can be effectively suppressed the proliferation of RA-FLS (Figure 2(a)). Subsequently, the intervention time among different groups indi- cated that the $\mathrm{OD}_{570}$ value in the high-dose group at $24 \mathrm{~h}$ was slightly decreased, while not obviously different compared with the $\mathrm{NC}$ group. However, the $\mathrm{OD}_{570}$ value of the high-dose group at $48 \mathrm{~h}$ and $72 \mathrm{~h}$ was significantly lower than those of the NC group and control serum group $(P<0.05)$ (Figure 2(b)). Those findings suggested that 20\% drugcontaining serum treated for $48 \mathrm{~h}$ was selected as the intervention conditions for the subsequent study.

3.3. Shentong Zhuyu Decoction Inhibited Viability and Promoted Apoptosis of RA-FLS. The results of flow cytometry showed that the apoptosis rate in the blank group was lower than that in the normal group $(P<0.05)$. In addition, the apoptosis rates in the P38 inhibitor group, PPAR $\gamma$ agonist group, and Shentong Zhuyu Decoction group were remarkably increased compared with the blank group $(P<0.05)$ (Figures 3(a) and 3(b)). Moreover, CCK-8 assay results indicated that the trend of the $\mathrm{OD}_{570}$ value was contrary to that of the apoptosis rate (Figure 3(c)). All data of qRT-PCR and 

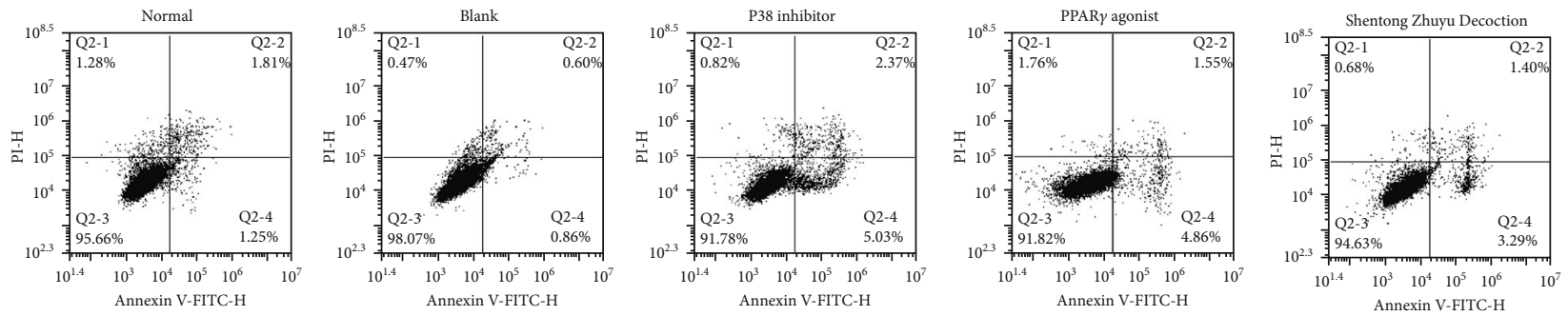

(a)

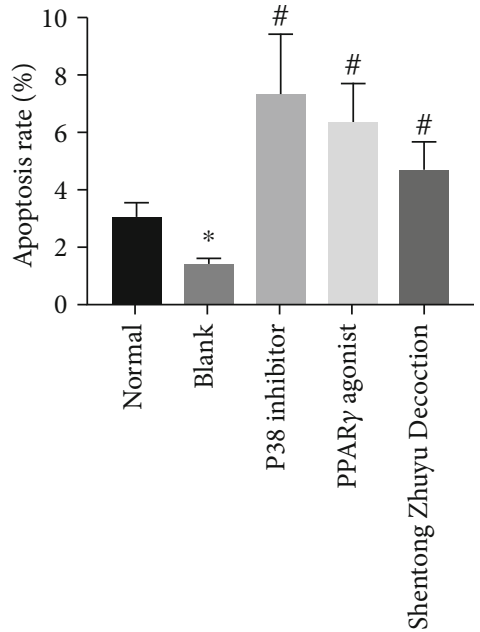

(b)

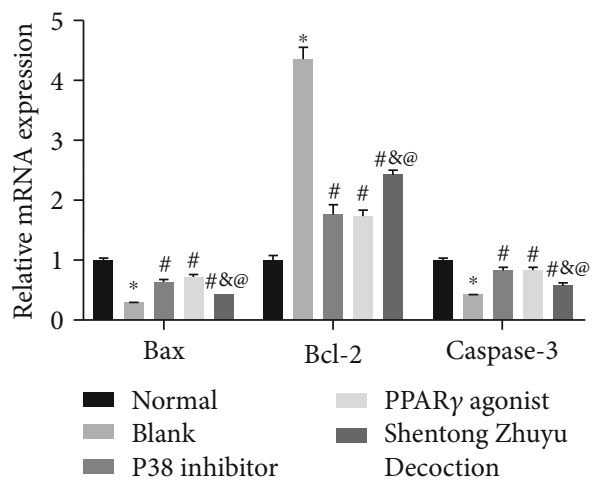

(d)

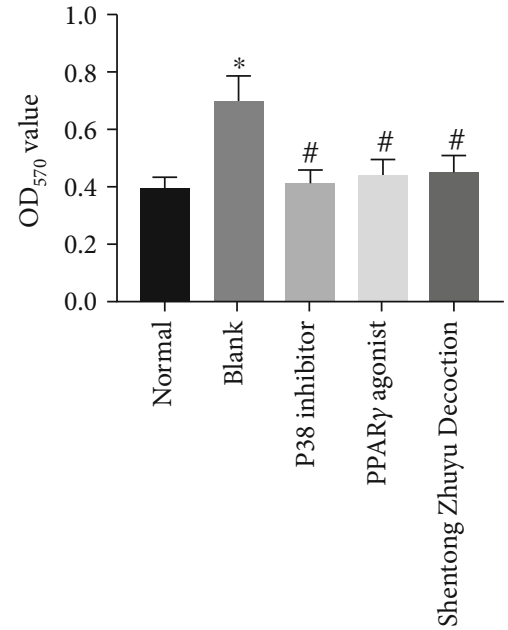

(c)

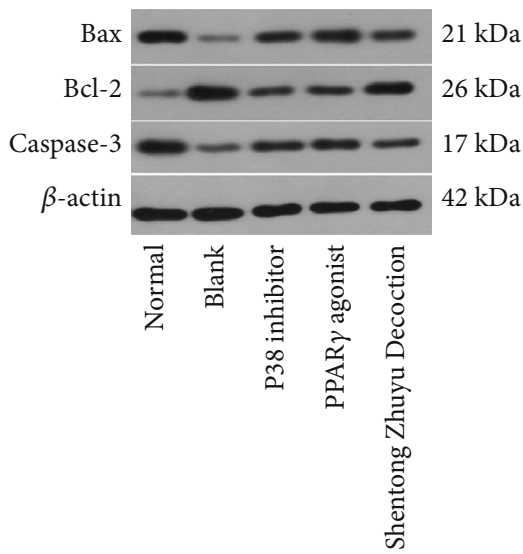

(e)

Figure 3: Continued. 


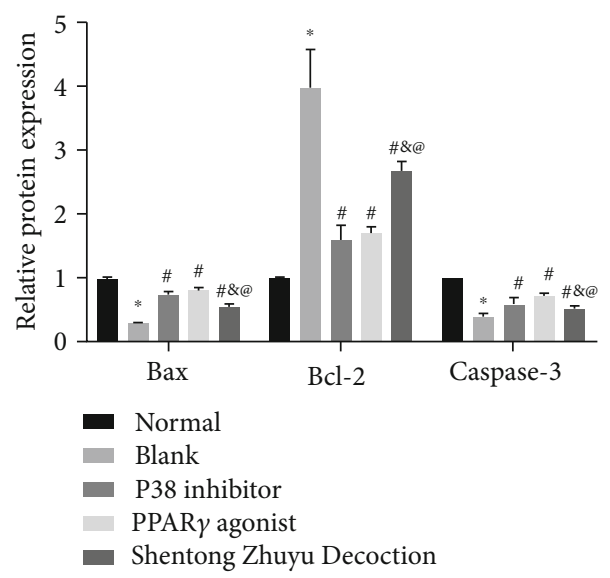

(f)

Figure 3: The effects of Shentong Zhuyu Decoction on RA-FLS. (a, b) The apoptosis rate of RA-FLS was detected by flow cytometry. (c) The $\mathrm{OD}_{570}$ value of RA-FLS was detected by the CCK-8 assay. (d) The mRNA levels of Bax, Bcl-2, and caspase-3 in RA-FLS were tested by qRTPCR. (e, f) The protein levels of Bax, Bcl-2, and caspase-3 in RA-FLS were tested by western blotting. ${ }^{*} P<0.05$ compared with the normal group, ${ }^{\#} P<0.05$ compared with the blank group, ${ }^{8} P<0.05$ compared with the P38 inhibitor group, and ${ }^{@} P<0.05$ compared with the PPAR $\gamma$ agonist group.

western blotting demonstrated that the levels of Bax and caspase-3 in the blank group were lower than those in the normal group $(P<0.05)$ and higher than in the P38 inhibitor group, PPAR $\gamma$ agonist group, and Shentong Zhuyu Decoction group $(P<0.05)$. Meanwhile, the expression of $\mathrm{Bcl}-2$ was opposite to that of Bax and caspase-3 (Figures 3(d)-3(f )). However, the $\mathrm{OD}_{570}$ value and apoptosis rate were not significant difference among the P38 inhibitor group, PPAR $\gamma$ agonist group, and Shentong Zhuyu Decoction group $(P>0.05)$.

3.4. Shentong Zhuyu Decoction Influenced the Cell Cycle of $R A-F L S$. As shown in Figures 4(a) and 4(b), the cell proportion in the $S$ phase was notably increased while the G1 phase was notably decreased in the blank group contrasted with the normal group (all $P<0.05$ ). In addition, the RA-FLS proportion in the $S$ phase was remarkably reduced, whereas significantly elevated in the G1 phase in the P38 inhibitor group, PPAR $\gamma$ agonist group, and Shentong Zhuyu Decoction group contrasted with the blank group $(P<0.05)$. However, cell proportion in the G2 phase was not obvious changes among different groups $(P>0.05)$. Furthermore, the results of qRTPCR and western blotting indicated that the levels of cyclin D1 and CDK 4 in the blank group were higher than those in the normal group $(P<0.05)$, whereas P38 inhibitor, $\operatorname{PPAR} \gamma$ agonist, and Shentong Zhuyu Decoction suppressed the expressions of cyclin D1 and CDK4 $(P<0.05)$. Among the treatment groups in RA-FLS, the effects of P38 inhibitor on the expression of cyclin D1 and CDK4 were equivalent to the PPAR $\gamma$ agonist $(P>0.05)$, while prior to the Shentong Zhuyu Decoction $(P<0.05)$ (Figures $4(\mathrm{c})-4(\mathrm{e}))$. All results suggested that Shentong Zhuyu Decoction inhibited the transition from the G1 phase to S phase of RA-FLS.

3.5. Shentong Zhuyu Decoction Inhibited Migration and Invasion of RA-FLS. As shown in Figures 5(a) and 5(b), the wound-healing rate in the blank group was faster than that in the normal group $(P<0.05)$ and slower than that in the P38 inhibitor group, PPAR $\gamma$ agonist group, and Shentong Zhuyu Decoction group $(P<0.05)$. Beyond that, the results of the transwell assay revealed that the numbers of invasive cells in the blank group were dramatically increased compared with the normal group, while were significantly decreased after intervened with P38 inhibitor, PPAR $\gamma$ agonist, and Shentong Zhuyu Decoction $(P<0.05)$ (Figures 5(c) and 5(d)). Of these, the impact of P38 inhibitor and PPAR $\gamma$ agonist on migration and invasion was stronger than Shentong Zhuyu Decoction $(P<0.05)$. Those findings suggested that Shentong Zhuyu Decoction inhibited the migration and invasion of RA-FLS.

3.6. Shentong Zhuyu Decoction Inhibited the Inflammatory Response of RA-FLS. The results showed that the levels of IL-1 $\beta$ and MMP-3 in the blank group were obviously increased compared with the normal group $(P<0.05)$. When intervened with P38 inhibitor, PPAR $\gamma$ agonist, and Shentong Zhuyu Decoction, the levels of IL- $1 \beta$ and MMP-3 were markedly reduced $(P<0.05)$. In addition, the effect of PPAR $\gamma$ agonist on the IL- $1 \beta$ expression was stronger than P38 inhibitor and Shentong Zhuyu Decoction $(P<0.05)$. However, the MMP-3 expression in the Shentong Zhuyu Decoction group was higher than that in the P38 inhibitor group and PPAR $\gamma$ agonist group $(P<0.05)$ (Figures $6(\mathrm{a})-$ $6(c))$.

3.7. Shentong Zhuyu Decoction Influenced the MAPK p38/PPAR $\gamma / C T G F$ Pathway. In order to explain the different effects of P38 inhibitor, PPAR $\gamma$ agonist, and Shentong Zhuyu Decoction on the RA-FLS, we investigated the expression of the MAPK p38/PPAR $\gamma / \mathrm{CTGF}$ pathway. As shown in Figures $7(\mathrm{a})-7(\mathrm{c})$, the mRNA and total protein levels of MAPK p38 were not obviously changes among the different groups $(P>0.05)$. Additionally, compared with the normal group, the levels of MAPK p-p38 and CTGF were markedly 

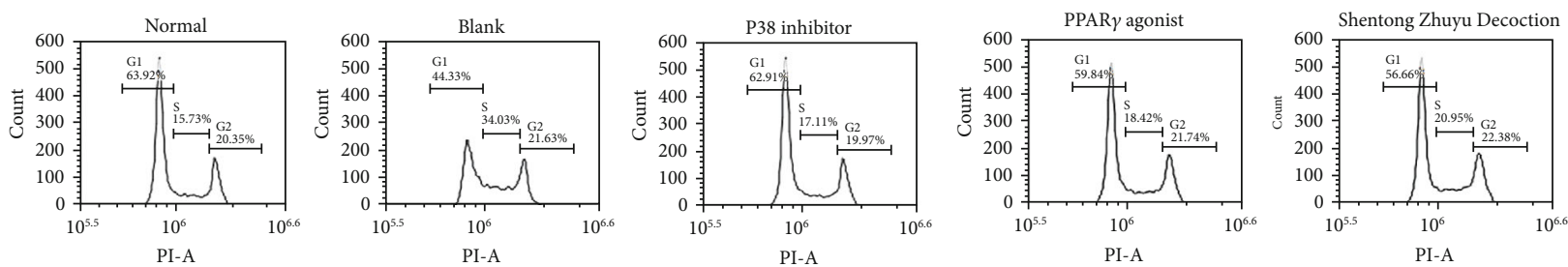

(a)
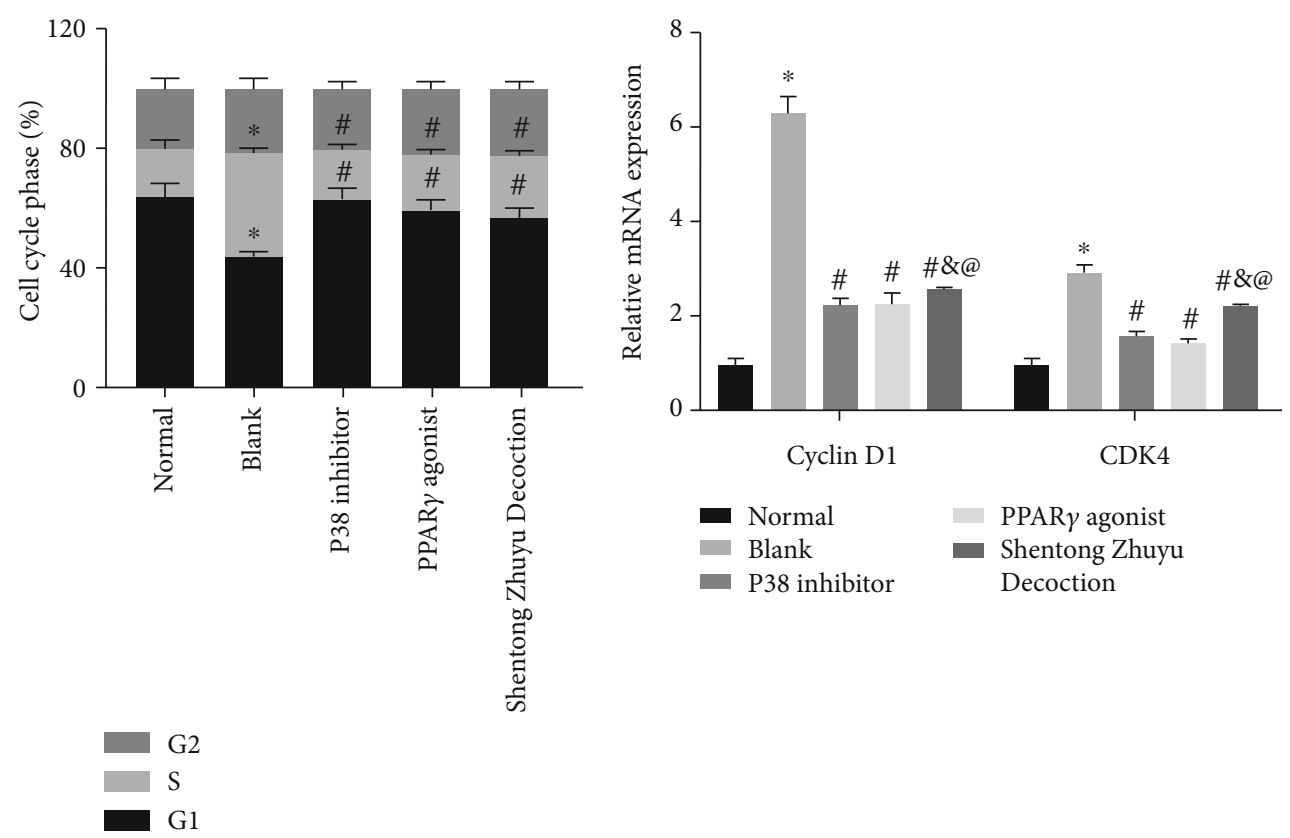

(b)

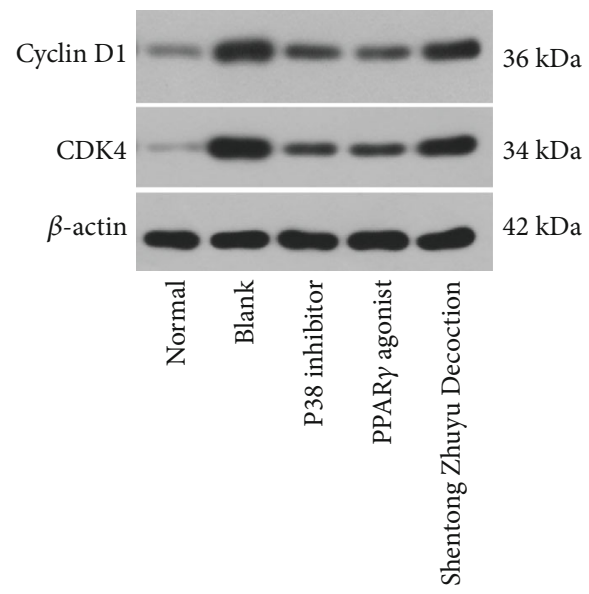

(d)

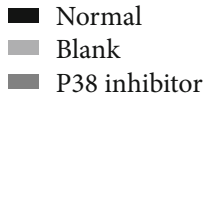

- PPAR $\gamma$ agonist

- Shentong Zhuyu

Decoction (c)

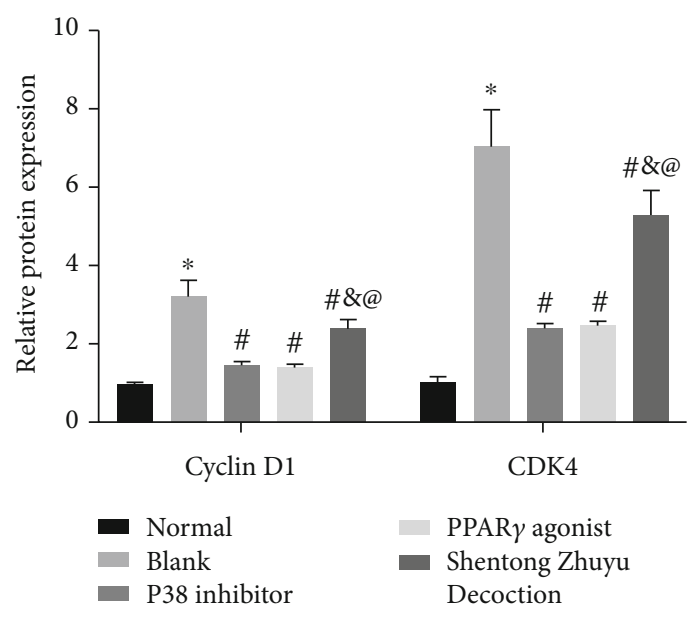

(e)

FIGURE 4: Shentong Zhuyu Decoction influenced the cell cycle of RA-FLS. (a, b) The cell cycle of RA-FLS was performed by flow cytometry. (c) The mRNA levels of cyclin D1 and CDK4 were tested by qRT-PCR. (d, e) The protein levels of cyclin D1 and CDK4 were tested by western blotting. ${ }^{*} P<0.05$ compared with the normal group, ${ }^{\#} P<0.05$ compared with the blank group; ${ }^{8} P<0.05$ compared with the P38 inhibitor group, and ${ }^{\circledR} P<0.05$ compared with the PPAR $\gamma$ agonist group.

elevated, while the PPAR $\gamma$ level was markedly reduced in the blank group $(P<0.05)$. Furthermore, P38 inhibitor, PPAR $\gamma$ agonist, and Shentong Zhuyu Decoction all can reverse the expression of MAPK p-p38, PPAR $\gamma$, and CTGF in the RAFLS $(P<0.05)$.
We also found that the MAPK p-p38 expression in the P38 inhibitor group was lower than that in the PPAR $\gamma$ agonist group. In terms of the PPAR $\gamma$ expression, the effect of PPAR $\gamma$ agonist was higher than P38 inhibitor, whereas the level of CTGF was not obviously different in the two groups. 


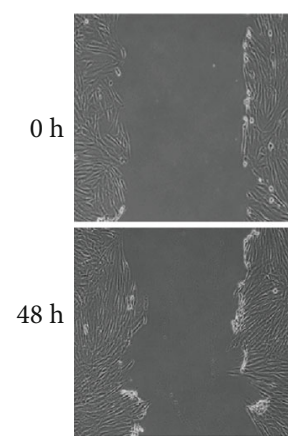

Normal

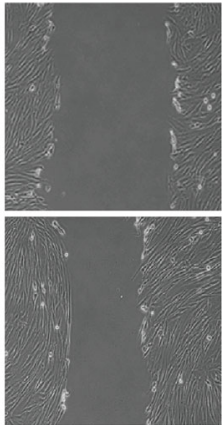

Blank

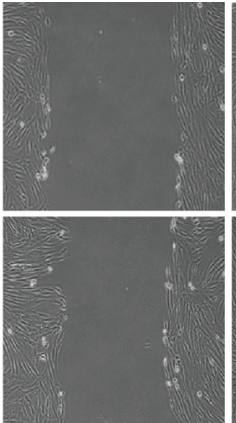

P38 inhibitor

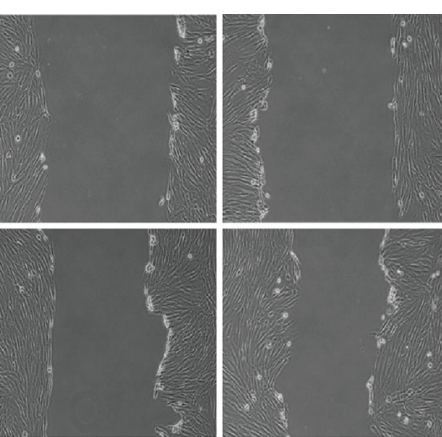

PPAR $\gamma$ agonist Shentong Zhuyu Decoction

(a)

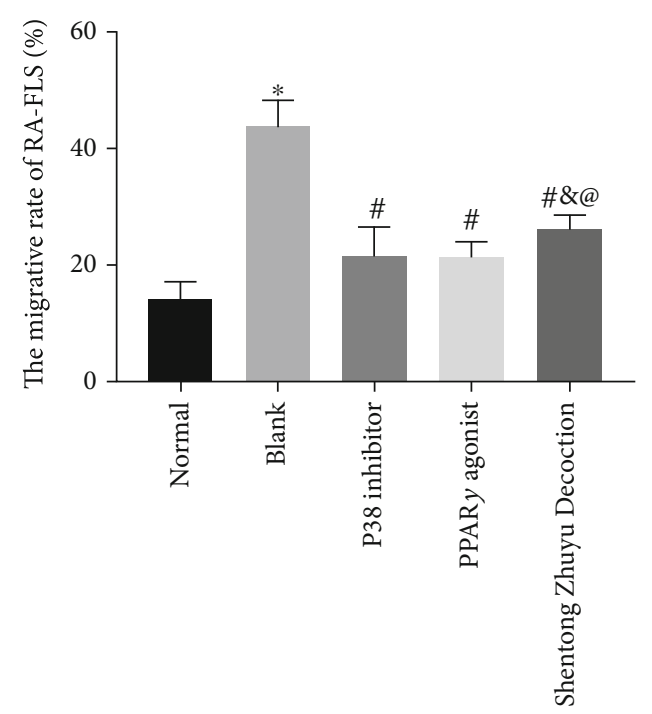

(b)

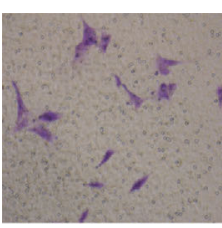

Normal

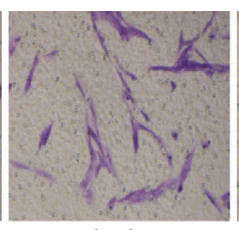

Blank

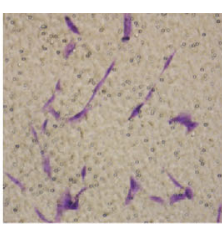

P38 inhibitor

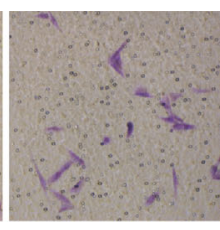

$\operatorname{PPAR} \gamma$ agonist

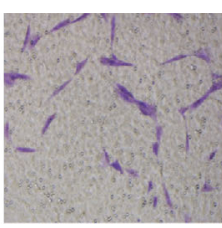

Shentong Zhuyu Decoction

(c)

Figure 5: Continued. 


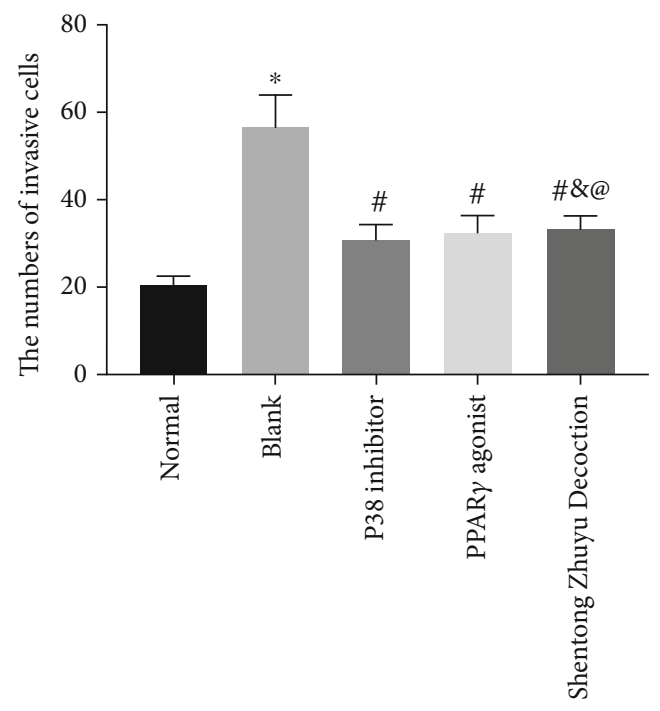

(d)

FIGURE 5: Shentong Zhuyu Decoction inhibited migration and invasion of RA-FLS. (a, b) The migration of RA-FLS was determined by the wound-healing assay $(\times 100)$. (c, d) The numbers of invasion were determined by the transwell assay $(\times 200)$. ${ }^{*} P<0.05$ compared with the normal group, ${ }^{\#} P<0.05$ compared with the blank group, ${ }^{\circledR} P<0.05$ compared with the P38 inhibitor group, and ${ }^{\circledR} P<0.05$ compared with the PPAR $\gamma$ agonist group.

So, we speculated that MAPK p-p38 may be upstream of PPAR $\gamma$, and CTGF may be jointly regulated by MAPK pp38 and PPAR $\gamma$. In addition, the levels of MAPK p-p38 and CTGF were significantly decreased, and the PPAR $\gamma$ level was notably elevated after intervention with Shentong Zhuyu Decoction in the RA-FLS (Figures $7(a)-7(\mathrm{c})$ ). Abovementioned results indicated that Shentong Zhuyu Decoction may inhibit proliferation, migration, and invasion and promote apoptosis in the RA-FLS via regulating the MAPK p38/PPAR $\gamma /$ CTGF pathway.

\section{Discussion}

RA pathogenesis is a systemic chronic inflammatory disease that is characterized as synovial cell proliferation with inflammatory infiltration, leading to joint damage [4]. RAFLS not only play the role in the inflammatory secretion but also manifest tumor-like phenotype with high proliferation, migration, and invasion [29]. So, targeting RA-FLS is important for treatment for RA. Currently, the drugs are available including methotrexate, glucocorticoids, and ibuprofen, which may cause some side effects. Beyond that, some biological drugs including tocilizumab and tofacitinib are expensive for clinical treatment [30]. In past decades, traditional Chinese medicine explains the pathogenesis of RA and holds the treatment on the basis of symptoms and signs $[20,31]$. Shentong Zhuyu Decoction is a classic representative formula for promoting blood circulation proposed by Qingren wang, which consists of 12 kinds of tradition Chinese herbs, including Gentiana macrophylla, Ligusticum wallichii, Peach Kernel, Safflower, Licorice, Notopterygium, Myrrh, Angelica, Trogopterus dung, Rhizoma Cyperi, Achyranthes, and Earthworm [21]. In the current study, the compound decoction added more 2 kinds of Chinese herbs on the basis of the above formula including Cortex phellodendri and Rhizoma Atractylodis to strengthen its power of dispelling wind, removing dampness, and clearing heat. Previous studies have proved that the combination of Cortex phellodendri and Rhizoma Atractylodis (Er-Miao-San) exhibits antiarthritic activity via regulating the balance of cytokines $[32,33]$. The major finding of our study was that Shentong Zhuyu Decoction can inhibit the inflammatory response, migration, and invasion and promote apoptosis of rheumatoid arthritis fibroblast-like synoviocytes via the MAPK p38/PPAR $\gamma /$ CTGF pathway.

Currently, induced by exogenous TNF- $\alpha$ and isolated from the synovial tissue are widely used for the RA model in vitro $[34,35]$. In our study, the CIA rat model was established, and then RA-FLS were isolated and identified, as described previously [36]. The current study showed that the cells cultured for 3 generations were in accordance with the characteristics of RA-FLS. Additionally, we also found that the abilities of proliferation, migration, and invasion in the RA-FLS were elevated, while the apoptosis rate was reduced compared with normal FLS. Furthermore, a previous study has indicated that inducing apoptosis is deemed as the ideal therapy for RA patients [37]. As we all known, cell cycle consists of G1, G2, and S phase (interphase), and $\mathrm{M}$ phase (mitosis), which closely related to the cell proliferation and apoptosis. Moreover, cyclin D1 and CDK4 form a protein kinase complex, which participates in the regulation of restriction points in the G1 phase and then promotes cells transited to the $S$ phase [38]. In terms of transition of the cell cycle in RA-FLS, Zhang et al. [39] have demonstrated that aspirin inhibits transition from the G1 phase to $S$ phase and then promotes apoptosis and inhibits proliferation. Surprisingly, our study demonstrated that Shentong Zhuyu Decoction can suppress the transition from the G1 phase to $S$ 


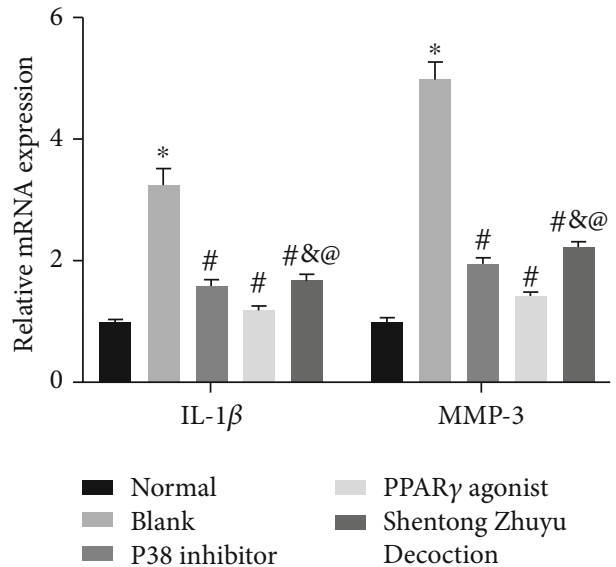

(a)

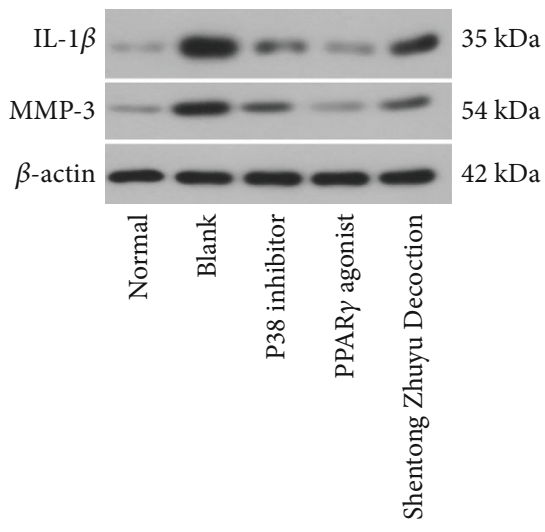

(b)

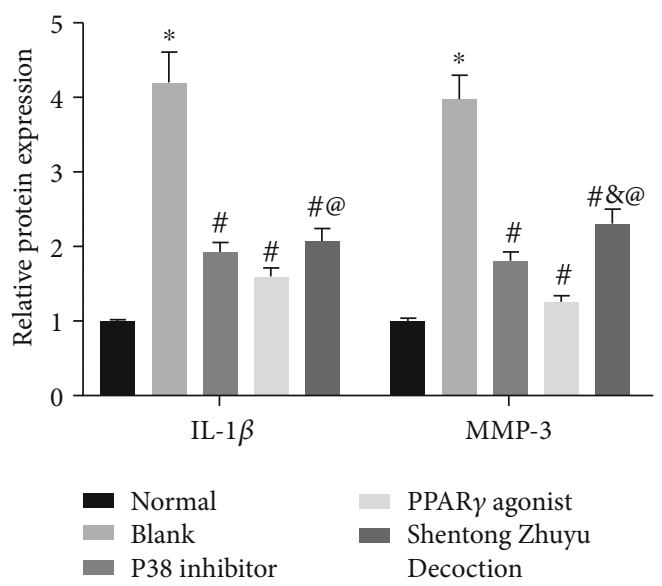

(c)

FIgURE 6: Shentong Zhuyu Decoction inhibited the inflammatory response of RA-FLS. (a) The mRNA levels of IL-1 $\beta$ and MMP3 were tested by qRT-PCR. (b, c) The protein levels of IL- $1 \beta$ and MMP-3 were tested by western blotting. ${ }^{*} P<0.05$ compared with the normal group, ${ }^{\#} P<0.05$ compared with the blank group, ${ }^{8} P<0.05$ compared with P38 inhibitor group, and ${ }^{\circledR} P<0.05$ compared with the PPAR $\gamma$ agonist group.

phase via inhibiting expressions of cyclin D1 and CDK4 in RA-FLS. Beyond that, we found that Shentong Zhuyu Decoction promoted the apoptosis rate of RA-FLS. In addition, the levels of $\mathrm{Bax}, \mathrm{Bcl}-2$, and caspase- 3 confirmed the results of the apoptosis. The proteins (Bax, $\mathrm{Bcl}-2$, and caspase-3) play crucial parts in apoptosis [31]. Abovementioned findings suggested that Shentong Zhuyu Decoction may become the targeting therapy for RA via regulating the cell biological behavior.

Inflammatory responses are the general event in RA patients, so antiinflammation therapy may be an effective treatment for relieving the symptoms [4]. It is well known that proinflammatory cytokines stimulate inflammatory responses and are considered as the key regulator in the progress of RA such as IL-1 $\beta$, TNF- $\alpha$, IL-6, and IL-17A [40]. In addition, these proinflammatory cytokines can promote the secretion of MMPs, which have been reported [41, 42]. Furthermore, Liu et al. [43] have indicated that Shentong Zhuyu Decoction can inhibit the secretion of inflammatory cytokines, attenuate cell infiltration, and improve state of joint. Surprisingly, the results of current study showed that the levels of IL- $1 \beta$ and MMP-3 were notably decreased after consumption of Shentong Zhuyu Decoction, which were consistent with previous report. Therefore, our study indicated that Shentong Zhuyu Decoction can effectively suppress the inflammatory response.

Until now, more evidences have verified that the MAPK signaling pathway involves in the regulation of inflammation and angiogenesis of RA [44-46] . Generally, MAPK p38 plays an important role on the secretion of proinflammatory cytokines [8]. A previous study has reported that MAPK p38 is upregulated and activated in RA-FLS [47]. Furthermore, Zou et al. have proved that SB203580 (p38 inhibitor) reverses the behaviors of activated RA-FLS [48]. Additionally, Ji et al. [49] have reported that pioglitazone (PPAR $\gamma$ agonist) suppresses inflammation via blocking the MAPK p38 pathway. Subsequently, a similar study has indicated that pioglitazone can effectively reduce the expression of MAPK p38 [50]. Our study showed that the MAPK p-p38 expression in the P38 inhibitor group was lower than that in the PPAR $\gamma$ agonist group, and the PPAR $\gamma$ expression of the PPAR $\gamma$ agonist group was higher than that of the P38 inhibitor group. So, 


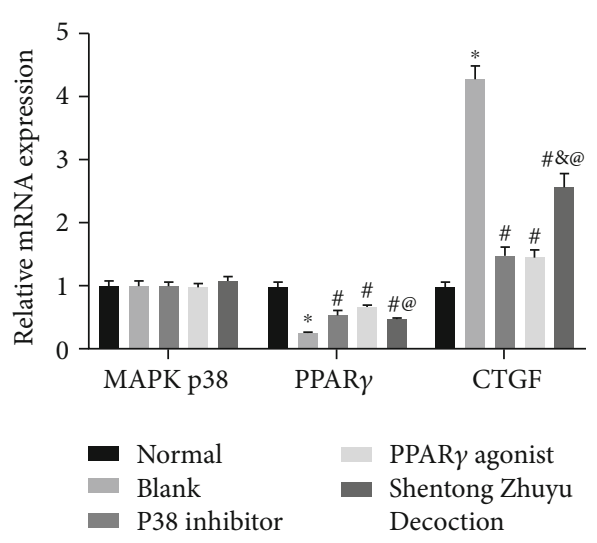

(a)

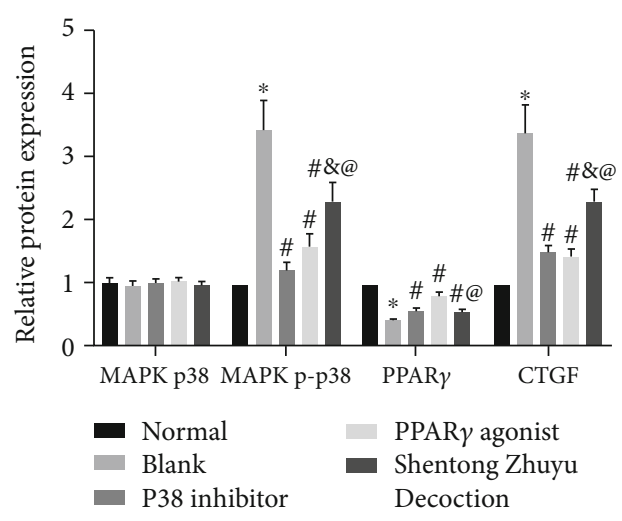

(c)

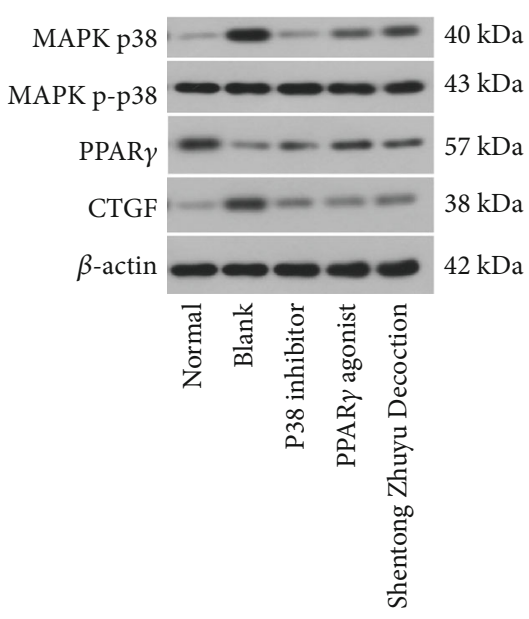

(b)

Figure 7: Shentong Zhuyu Decoction influenced the MAPK p38/PPAR $\gamma /$ CTGF pathway. (a) The mRNA levels of MAPK p38, PPAR $\gamma$, and CTGF were tested by qRT-PCR. (b, c) The protein levels of MAPK p38, MAPK p-p38, PPAR $\gamma$, and CTGF were tested by western blotting. ${ }^{*} P<0.05$ compared with the normal group, ${ }^{\#} P<0.05$ compared with the blank group, ${ }^{8} P<0.05$ compared with the P38 inhibitor group, ${ }^{\circledR} P<0.05$ compared with the PPAR $\gamma$ agonist group.

we speculated that MAPK p38 may be upstream of PPAR $\gamma$, and there is a negative regulation relationship. CTGF is regarded as a novel mediator for cell growth, which was secreted by fibroblasts [51]. Apart from silencing of MAPK p38, it significantly decreases the CTGF expression, while PPAR $\gamma$ agonist reduced the CTGF level [52, 53]. In our study, the CTGF level was not obviously different between the P38 inhibitor group and PPAR $\gamma$ agonist group. Those findings indicated that CTGF may be jointly regulated by MAPK p-p38 and PPAR $\gamma$. Furthermore, we also found that Shentong Zhuyu Decoction inhibited the levels of MAPK p-p38 and CTGF and promoted the PPAR $\gamma$ level. The results of Shentong Zhuyu Decoction were consistent with the trends of SB203580 and GW1929. Therefore, abovementioned results suggested that Shentong Zhuyu Decoction regulated the behaviors of RA-FLS via the MAPK p38/PPAR $\gamma /$ CTGF pathway.

The lack of research on Shentong Zhuyu Decoction in vitro is a limitation of this study. So, we will explore the effect of Shentong Zhuyu Decoction on the CIA model and verify the effect of drug-containing serum via administration of Shentong Zhuyu Decoction in vivo in the further.

\section{Conclusions}

The current study demonstrated that Shentong Zhuyu Decoction inhibited inflammatory response, transition from G1 phase to $S$ phase, migration, and invasion and promoted apoptosis of RA-FLS via regulating the MAPK p38/PPAR $\gamma / \mathrm{CTGF}$ pathway.

\section{Data Availability}

All data generated or analyzed in this study are included in this published article.

\section{Conflicts of Interest}

All authors have no potential conflicts of interest.

\section{Acknowledgments}

The study was funded by the Research on Weiqi Rhythm and Prescription Intervention Based on Metabonomics (GZR 81273609). 


\section{References}

[1] A. I. Catrina, C. I. Svensson, V. Malmstrom, G. Schett, and L. Klareskog, "Mechanisms leading from systemic autoimmunity to joint-specific disease in rheumatoid arthritis," Nature Reviews Rheumatology, vol. 13, no. 2, pp. 79-86, 2017.

[2] G. S. Firestein and I. B. McInnes, "Immunopathogenesis of rheumatoid arthritis," Immunity, vol. 46, no. 2, pp. 183-196, 2017.

[3] P. P. Tak and J. R. Kalden, "Advances in rheumatology: new targeted therapeutics," Arthritis Research \& Therapy, vol. 13, Supplement 1, p. S5, 2011.

[4] J. S. Smolen, D. Aletaha, and I. B. McInnes, "Rheumatoid arthritis," The Lancet, vol. 388, no. 10055, pp. 2023-2038, 2016.

[5] M. C. Lebre, P. L. Vieira, M. W. Tang et al., "Synovial IL21/TNF-producing $\mathrm{CD}^{+}{ }^{+} \mathrm{T}$ cells induce joint destruction in rheumatoid arthritis by inducing matrix metalloproteinase production by fibroblast-like synoviocytes," Journal of Leukocyte Biology, vol. 101, no. 3, pp. 775-783, 2017.

[6] A. Korb-Pap, J. Bertrand, J. Sherwood, and T. Pap, "Stable activation of fibroblasts in rheumatic arthritis-causes and consequences," Rheumatology, vol. 55, Supplement 2, pp. ii64-ii67, 2016.

[7] A. Filer, "The fibroblast as a therapeutic target in rheumatoid arthritis," Current Opinion in Pharmacology, vol. 13, no. 3, pp. 413-419, 2013.

[8] G. L. Johnson and R. Lapadat, "Mitogen-activated protein kinase pathways mediated by ERK, JNK, and p38 protein kinases," Science, vol. 298, no. 5600, pp. 1911-1912, 2002.

[9] M. H. Tsai, M. C. Chi, and J. F. Hsu, "Urban particulate matter enhances ROS/IL-6/COX-II production by inhibiting microRNA-137 in synovial fibroblast of rheumatoid arthritis," Cell, vol. 9, no. 6, p. 1378, 2020.

[10] H. du, X. Zhang, Y. Zeng et al., "A novel phytochemical, DIM, inhibits proliferation, migration, invasion and TNF- $\alpha$ induced inflammatory cytokine production of synovial fibroblasts from rheumatoid arthritis patients by targeting MAPK and AKT/mTOR signal pathway," Frontiers in Immunology, vol. 10, p. 1620, 2019.

[11] T. H. Lin, C. H. Tang, K. Wu, Y. C. Fong, R. S. Yang, and W. M. Fu, "15-deoxy- $\Delta(12,14)$-prostaglandin-J2 and ciglitazone inhibit TNF- $\alpha$-induced matrix metalloproteinase 13 production via the antagonism of NF- $\kappa \mathrm{B}$ activation in human synovial fibroblasts," Journal of Cellular Physiology, vol. 226, pp. 3242-3250, 2011.

[12] S. W. Lee, J. Y. Won, W. J. Kim et al., "Snail as a potential target molecule in cardiac fibrosis: paracrine action of endothelial cells on fibroblasts through snail and CTGF axis," Molecular Therapy, vol. 21, no. 9, pp. 1767-1777, 2013.

[13] W. Sun, J. Ma, H. Zhao et al., "Resolvin D1 suppresses pannus formation via decreasing connective tissue growth factor caused by upregulation of miRNA-146a-5p in rheumatoid arthritis," Arthritis Research \& Therapy, vol. 22, no. 1, p. 61, 2020.

[14] C. Tian, X. Jin, X. Ye et al., "Long term intake of $0.1 \%$ ethanol decreases serum adiponectin by suppressing PPAR $\gamma$ expression via p38 MAPK pathway," Food and Chemical Toxicology, vol. 65, pp. 329-334, 2014.

[15] K. F. Zhai, H. Duan, C. Y. Cui et al., "Liquiritin from glycyrrhiza uralensis attenuating rheumatoid arthritis via reducing inflammation, suppressing angiogenesis, and inhibiting
MAPK signaling pathway," Journal of Agricultural and Food Chemistry, vol. 67, no. 10, pp. 2856-2864, 2019.

[16] H. Wang, S. Tu, S. Yang et al., "Berberine modulates LPA function to inhibit the proliferation and inflammation of FLS-RA via p38/ERK MAPK pathway mediated by LPA1," Evidencebased Complementary and Alternative Medicine, vol. 2019, Article ID 2580207, 12 pages, 2019.

[17] K. F. Zhai, H. Duan, G. J. Khan et al., "Salicin from Alangium chinense ameliorates rheumatoid arthritis by modulating the Nrf2-HO-1-ROS pathways," Journal of Agricultural and Food Chemistry, vol. 66, no. 24, pp. 6073-6082, 2018.

[18] K. F. Zhai, H. Duan, Y. Chen et al., "Apoptosis effects of imperatorin on synoviocytes in rheumatoid arthritis through mitochondrial/caspase-mediated pathways," Food \& Function, vol. 9, no. 4, pp. 2070-2079, 2018.

[19] K. F. Zhai, H. Duan, L. Luo et al., "Protective effects of paeonol on inflammatory response in IL- $1 \beta$-induced human fibroblast-like synoviocytes and rheumatoid arthritis progression via modulating NF- $\kappa \mathrm{B}$ pathway," Inflammopharmacology, vol. 25, no. 5, pp. 523-532, 2017.

[20] H. Duan, K. F. Zhai, G. J. Khan et al., "Revealing the synergistic mechanism of multiple components in compound Fengshiding capsule for rheumatoid arthritis therapeutics by network pharmacology," Current Molecular Medicine, vol. 19, no. 4, pp. 303-314, 2019.

[21] X. J. Lin and C. Y. Chen, "Advances on study of treatment of lumbar disk herniation by Chinese medicinal herbs," Zhongguo Zhong Yao Za Zhi, vol. 32, no. 3, pp. 186-191, 2007.

[22] B. X. Ren, Z. L. Ma, and Y. Q. Jin, "Effect of shentong zhuyu decoction on pain behavior and spinal cord astrocytes model of osteocarcinoma pain," Zhongguo Zhong Xi Yi Jie He Za Zhi, vol. 31, no. 3, pp. 381-385, 2011.

[23] K. Sun, L.-G. Zhu, X. Wei et al., "Efficacy and safety of Shentong Zhuyu Decoction for lumbar disc herniation: systematic review and meta-analysis," Zhongguo Zhong Yao Za Zhi, vol. 45, pp. 1159-1166, 2020.

[24] J. Pu, F. F. Fang, X. Q. Li et al., "Matrine exerts a strong antiarthritic effect on type II collagen-induced arthritis in rats by inhibiting inflammatory responses," International Journal of Molecular Sciences, vol. 17, no. 9, p. 1410, 2016.

[25] E. F. Rosloniec, M. Cremer, A. H. Kang, L. K. Myers, and D. D. Brand, "Collagen-induced arthritis," Current Protocols in Immunology, vol. 89, no. 1, 2010.

[26] M. A. Lim, B. Louie, D. Ford et al., "Development of the digital arthritis index, a novel metric to measure disease parameters in a rat model of rheumatoid arthritis," Frontiers in Pharmacology, vol. 8, p. 818, 2017.

[27] L. W. Chou, J. Wang, P. L. Chang, and Y. L. Hsieh, "Hyaluronan modulates accumulation of hypoxia-inducible factor-1 alpha, inducible nitric oxide synthase, and matrix metalloproteinase-3 in the synovium of rat adjuvant-induced arthritis model," Arthritis Research \& Therapy, vol. 13, no. 3, p. R90, 2011.

[28] K. F. Zhai, J. R. Zheng, Y. M. Tang et al., "The saponin D39 blocks dissociation of non-muscular myosin heavy chain IIA from TNF receptor 2, suppressing tissue factor expression and venous thrombosis," British Journal of Pharmacology, vol. 174, no. 17, pp. 2818-2831, 2017.

[29] L. C. Huber, O. Distler, I. Tarner, R. E. Gay, S. Gay, and T. Pap, "Synovial fibroblasts: key players in rheumatoid arthritis," Rheumatology, vol. 45, no. 6, pp. 669-675, 2006. 
[30] D. Aletaha and J. S. Smolen, "Diagnosis and management of rheumatoid arthritis," JAMA, vol. 320, no. 13, pp. 13601372, 2018.

[31] A. Zhang, Y. Wu, H. W. Lai, and D. T. Yew, "Apoptosis-a brief review," Neuroembryology and Aging, vol. 3, pp. 47-59, 2005.

[32] G. Chen, K. K. Li, C. H. Fung et al., "Er-Miao-San, a traditional herbal formula containing Rhizoma Atractylodis and Cortex Phellodendri inhibits inflammatory mediators in LPSstimulated RAW264.7 macrophages through inhibition of NF- $\kappa$ B pathway and MAPKs activation," Journal of Ethnopharmacology, vol. 154, no. 3, pp. 711-718, 2014.

[33] X. Dai, D. Yang, J. Bao et al., "Er Miao San, a traditional Chinese herbal formula, attenuates complete Freund's adjuvantinduced arthritis in rats by regulating Th17/Treg cells," Pharmaceutical Biology, vol. 58, no. 1, pp. 157-164, 2020.

[34] Q. Zhang, W. Peng, S. Wei et al., "Guizhi-Shaoyao-Zhimu decoction possesses anti-arthritic effects on type II collageninduced arthritis in rats _via_ suppression of inflammatory reactions, inhibition of invasion \& migration and induction of apoptosis in synovial fibroblasts," Biomedicine \& Pharmacotherapy, vol. 118, article 109367, 2019.

[35] K. Chen, Z. W. Lin, S. M. He et al., "Metformin inhibits the proliferation of rheumatoid arthritis fibroblast-like synoviocytes through IGF-IR/PI3K/AKT/m-TOR pathway," Biomedicine \& Pharmacotherapy, vol. 115, article 108875, 2019.

[36] M. R. Tanner, M. W. Pennington, S. S. Chauhan, T. Laragione, P. S. Gulko, and C. Beeton, "KCa1.1 and Kv1.3 channels regulate the interactions between fibroblast-like synoviocytes and $\mathrm{T}$ lymphocytes during rheumatoid arthritis," Arthritis Research \& Therapy, vol. 21, no. 1, p. 6, 2019.

[37] I. B. Mc Innes and G. Schett, "Pathogenetic insights from the treatment of rheumatoid arthritis," The Lancet, vol. 389, no. 10086, pp. 2328-2337, 2017.

[38] J. P. Matson and J. G. Cook, "Cell cycle proliferation decisions: the impact of single cell analyses," The FEBS Journal, vol. 284, no. 3, pp. 362-375, 2017.

[39] X. Zhang, H. Feng, J. du et al., “Aspirin promotes apoptosis and inhibits proliferation by blocking G0/G1 into S phase in rheumatoid arthritis fibroblast-like synoviocytes via downregulation of JAK/STAT3 and NF- $\kappa \mathrm{B}$ signaling pathway," International Journal of Molecular Medicine, vol. 42, pp. 31353148, 2018.

[40] K. Chaabo and B. Kirkham, "Rheumatoid Arthritis - AntiTNF,” International Immunopharmacology, vol. 27, pp. 180184, 2015.

[41] C. E. Page, S. Smale, S. M. Carty et al., "Interferon-gamma inhibits interleukin-1beta-induced matrix metalloproteinase production by synovial fibroblasts and protects articular cartilage in early arthritis," Arthritis Research \& Therapy, vol. 12, p. R49, 2010.

[42] C. Angiolilli, A. M. Grabiec, B. S. Ferguson et al., "Inflammatory cytokines epigenetically regulate rheumatoid arthritis fibroblast-like synoviocyte activation by suppressing HDAC5 expression," Annals of the Rheumatic Diseases, vol. 75, no. 2, pp. 430-438, 2016.

[43] J. Liu and Y. Sun, "How does Chinese medicine target cytokine imbalance in rheumatoid arthritis," Chinese Journal of Integrative Medicine, vol. 19, no. 11, pp. 874-880, 2013.

[44] K. F. Zhai, H. Duan, C. Y. Cui et al., "Liquiritin fromGlycyrrhiza uralensisattenuating rheumatoid arthritis via reducing inflammation, suppressing angiogenesis, and inhibiting
MAPK signaling pathway," Journal of Agricultural and Food Chemistry, vol. 67, no. 10, pp. 2856-2864, 2019.

[45] L. Zhang, J. Luo, H. Wen, T. Zhang, X. Zuo, and X. Li, “MDM2 promotes rheumatoid arthritis via activation of MAPK and $\mathrm{NF}-\kappa \mathrm{B}, "$ International Immunopharmacology, vol. 30, pp. 69-73, 2016.

[46] L. Xu, X. Feng, Y. Shi et al., "Interleukin-29 induces receptor activator of NF- $\kappa$ B ligand expression in fibroblast-like synoviocytes via MAPK signaling pathways," International Journal of Rheumatic Diseases, vol. 18, no. 8, pp. 842-849, 2015.

[47] L. Liang, M. Huang, Y. Xiao et al., "Inhibitory effects of niclosamide on inflammation and migration of fibroblast-like synoviocytes from patients with rheumatoid arthritis," Inflammation Research, vol. 64, no. 3-4, pp. 225-233, 2015.

[48] Q. F. Zou, L. Li, Q. R. Han, Y. J. Wang, and X. B. Wang, “Abatacept alleviates rheumatoid arthritis development by inhibiting migration of fibroblast-like synoviocytes via MAPK pathway," European Review for Medical and Pharmacological Sciences, vol. 23, pp. 3105-3111, 2019.

[49] H. Ji, H. Wang, F. Zhang, X. Li, L. Xiang, and S. Aiguo, "PPAR $\gamma$ agonist pioglitazone inhibits microglia inflammation by blocking p38 mitogen-activated protein kinase signaling pathways," Inflammation Research, vol. 59, pp. 921-929, 2010.

[50] J. Li, B. Xu, Z. Chen et al., "PI3K/AKT/JNK/p38 signalling pathway-mediated neural apoptosis in the prefrontal cortex of mice is involved in the antidepressant-like effect of pioglitazone," vol. 45, pp. 525-535, 2018.

[51] P. Bork, "The modular architecture of a new family of growth regulators related to connective tissue growth factor," FEBS Letters, vol. 327, pp. 125-130, 1993.

[52] W. Wang, F. Liu, and N. Chen, "Peroxisome proliferatoractivated receptor- $\gamma$ (PPAR- $\gamma$ ) agonists attenuate the profibrotic response induced by TGF- $\beta 1$ in renal interstitial fibroblasts," Mediators of Inflammation, vol. 2007, Article ID 62641, 7 pages, 2007.

[53] Y. L. Cao, Y. Duan, L. X. Zhu, Y. N. Zhan, S. X. Min, and A. M. Jin, "TGF- $\beta 1$, in association with the increased expression of connective tissue growth factor, induce the hypertrophy of the ligamentum flavum cthrough the p38 MAPK pathway," International Journal of Molecular Medicine, vol. 38, no. 2, pp. 391-398, 2016. 